\title{
Neuroplasticity and pain: what does it all mean?
}

\author{
Recent findings have implications for how we conceptualise, assess and treat pain
}

\section{Philip J Siddall MB BS, PhD, FFPMANZCA Pain Medicine Physician, and Professor in Pain Medicine $^{2}$ \\ 1 Greenwich Hospital, HammondCare, Sydney, NSW. \\ 2 Northern Clinical School, University of Sydney, Sydney, NSW. \\ phil.siddall@ sydney.edu.au}

doi: 10.5694/mjal3.10100

Philip Siddall will be presenting at the Australian Pain Society Annual Scientific eeting in Canberra,

17-20 March 2013

(http://www.apsoc.org.au/ conference)

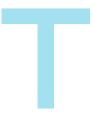

he concept of neuroplasticity - the ability of the nervous system to change its structure and function - has captured the imagination of clinicians, researchers and the general public. ${ }^{1}$ The ability of the brain to reorganise itself is remarkable. For example, people who are visually impaired engage their visual cortex for fine sensory discrimination when using their hands. In these situations, neuroplasticity appears to be a positive adaptation to loss of function.

Recent neuroplasticity studies have shown that pain is associated with a host of functional, anatomical and chemical changes at many levels of the nervous system. ${ }^{2,3}$ The most intriguing and dramatic example of functional changes is the reorganisation that occurs at a cortical level, particularly in association with major trauma to the nervous system such as limb amputation or spinal cord injury. ${ }^{4,5}$ In these situations, it has been shown that regions of the cortical homunculus that normally respond to inputs from a part of the body that has been denervated can be activated by stimuli that would normally activate an adjacent region of the sensory cortex. For example, stimulation of the lip in people with an amputated arm will activate the lip region as well as the adjacent sensory cortex normally activated by inputs from the hand. This reorganisation has been shown to be important in pain medicine. Although the direction of causality is unclear, the reorganisation is strongly linked to the presence of pain. Therefore, neuroplasticity may be an attempt by the nervous system to adapt to injury in a positive way. But in the case of pain, neuroplasticity appears to be maladaptive.

Chemical and structural changes which occur in the presence of pain and injury include receptor and neurotransmitter changes that flow through to physiological phenomena such as alterations in pain modulation ${ }^{6,7}$ and sensitisation of central neurones involved in the transmission of nociceptive signals. ${ }^{3,8}$ These changes amplify signals arising from tissue damage and can increase the intensity of experienced pain.

Understanding neuroplasticity has put paid to the old concept that pain is merely another sensation transmitted along hard-wired pathways. Findings regarding the complexity of pain processing, including neuroplasticity, show that the experience of pain engages an entire orchestra of pathophysiology. This means that the best chance of success in treating pain occurs when assessment and treatment address these many factors. For example, treat- ing persistent low back pain means not just trying to determine which structures in the back may be contributing to pain - it also means identifying any psychological factors that may alter the responsiveness of the nervous system and amplify incoming signals.

However, these findings provide reasons for hope. The first reason is that, although it may sound strange, neuroplasticity is by nature plastic. ${ }^{9}$ This means that although nervous system changes can occur, they are not necessarily irreversible. Some authors have adopted the view that central processes can become so dominant and fixed that they generate pain in the absence of peripheral input. Fortunately, there is not a great deal of evidence to support this view. Although nervous system injury such as damage to the spinal cord may give rise to structural changes that are extremely resistant to change, activity-dependent neuroplasticity (central changes that are dependent on the level of incoming signals to the spinal cord and brain) in response to nociceptive inputs is not so resistant. Clinically, this is seen by the large number of people with osteoarthritis of the hip who experience relief of pain following total hip replacement, no matter how severe the pain or how long it has been present. It has been shown that people who have pain associated with osteoarthritis of the hip have neuroplastic changes, including a reduction in grey matter volume in some brain regions. Importantly, these changes have been shown to reverse following successful hip surgery and a decrease in pain. ${ }^{10}$ This suggests that neuroplasticity is dependent on pain rather than the other way around.

The other reason for hope is that the findings provide us with a much better understanding of the role of psychological factors in pain and the potential for treatment. There is no room for the old dualist view of pain being either real or psychological. Cognitive and emotional processes strongly engage brain and spinal cord pathways that are directly involved in altering the responsiveness of pain pathways. By doing so, psychological factors influence neuroplastic processes and thus directly modify the pain experience. $^{7}$

The role of mood and thought processes in modifying neuroplastic changes builds further support for the role of psychological interventions in treating pain, not just as a way of being able to cope with pain, but as an important and effective option for relieving pain. 
Effective pain management therefore relies on judicious use of treatments that, as far as possible, reduce inputs from the periphery, and on the wide and growing range of pharmacological, physical and psychological approaches that are known to modify central pain processing and reduce pain.

Neuroplasticity research has opened our eyes to a whole new world of mechanisms underlying pain and given us a greater appreciation of the complex interaction between the mind and body. It has provided important keys that hold promise and hope for the future.

Acknowledgements: I currently receive funding from National Health and Medical Research Council and Australian and New Zealand College of Anaesthetists grants.

Competing interests: No relevant disclosures.

Provenance: Commissioned; externally peer reviewed.

1 Doidge $N$. The brain that changes itself: stories of personal triumph from the frontiers of brain science. London: Penguin Books, 2007.
2 Seifert F, Maihofner C. Functional and structural imaging of pain-induced neuroplasticity. Curr Opin Anaesthesiol 2011; 24: 515-523.

3 Latremoliere A, Woolf CJ. Central sensitization: a generator of pain hypersensitivity by central neural plasticity. J Pain 2009; 10: 895-926.

4 Flor H, Elbert T, Knecht S, et al. Phantom-limb pain as a perceptual correlate of cortical reorganization following arm amputation. Nature 1995; 375: 482-484.

5 Wrigley PJ, Press SR, Gustin SM, et al. Neuropathic pain and primary somatosensory cortex reorganization following spinal cord injury. Pain 2009; 141: 52-59.

6 Melzack R, Coderre TJ, Katz J, Vaccarino AL. Central neuroplasticity and pathological pain. Ann N Y Acad Sci 2001; 933: 157-174.

7 Price DD, Verne GN, Schwartz JM. Plasticity in brain processing and modulation of pain. Prog Brain Res 2006; 157: 333-352.

8 Sandkühler J. Learning and memory in pain pathways. Pain 2000; 88: 113-118.

9 Knecht S, Henningsen $\mathrm{H}$, Hohling C, et al. Plasticity of plasticity - changes in the pattern of perceptual correlates of reorganization after amputation. Brain 1998; 121: 717-724.

10 Gwilym SE, Filippini N, Douaud G, et al. Thalamic atrophy associated with painful osteoarthritis of the hip is reversible after arthroplasty: a longitudinal voxel-based morphometric study. Arthritis Rheum 2010; 62: 2930-2940. 